\title{
Hybrid intelligent diagnosis approach based on soft computing from signal and image knowledge representations for a biomedical application
}

\author{
Amine Chohra • Nadia Kanaoui · \\ Véronique Amarger • Kurosh Madani
}

Received: 2 December 2013 / Accepted: 20 February 2014 / Published online: 23 March 2014

(C) The Author(s) 2014. This article is published with open access at Springerlink.com

\begin{abstract}
Fault diagnosis is a complex and fuzzy cognitive process, and soft computing methods as neural networks and fuzzy logic, have shown great potential in the development of decision support systems. Dealing with expert (human) knowledge consideration, Computer-Aided Diagnosis (CAD) dilemma is one of the most interesting, but also one of the most difficult problems. Among difficulties contributing to challenging nature of this problem, one can mention the need of fine classification and decision-making. In this paper, a brief survey on fault diagnosis systems is given first. Then, from a fault diagnosis system analysis of the classification and decision-making problem, a global diagnosis synopsis is deduced. Afterwards, a hybrid intelligent diagnosis approach, based on soft computing implying modular neural networks for classification and fuzzy logic for decision-making, is suggested from signal and image representations. The suggested approach is developed in biomedicine for a CAD, from Auditory Brainstem Response test, and the prototype design and experimental results are presented. In fact, a double classification is exploited in a primary fuzzy diagnosis, to ensure a satisfactory reliability. Then, this reliability is reinforced using a confidence parameter with the primary diagnosis result, exploited in a final fuzzy diagnosis giving the appropriate diagnosis with a

\author{
A. Chohra $(\bowtie) \cdot$ N. Kanaoui · V. Amarger · K. Madani \\ Images, Signals, and Intelligent Systems Laboratory (LISSI / EA 3956), \\ Senart Institute of Technology, Paris East University (UPEC), \\ Avenue Pierre Point, 77127 Lieusaint, France \\ e-mail: chohra@u-pec.fr \\ N. Kanaoui \\ e-mail: kanaoui@u-pec.fr \\ V. Amarger \\ e-mail: amarger@u-pec.fr \\ K. Madani \\ e-mail: madani@u-pec.fr
}

confidence index. Indeed, experimental results demonstrate the efficiency and reliability of CAD for three classes: two auditory pathologies Retro-cochlear Class (RC) and Endocochlear Class (EC), and Normal auditory Class (NC). The generalization rate of $\mathrm{NC}$ is clearly higher for primary fuzzy diagnosis and final fuzzy diagnosis than that of the two classifications. The obtained rates for RC and EC are higher than obtained by image classification but quite similar than those obtained by signal classification. An important contribution of the final fuzzy diagnosis is the fact that a confidence index is associated with each fault diagnosis. Finally, a discussion is given with regard to the reliability and large application field of the suggested approach.

Keywords Decision support - Classification . Decision-making $\cdot$ Learning and adaptation .

Neural networks · Fuzzy logic

\section{Introduction}

A diagnosis system is basically one which is capable of identifying the nature of a problem by examining the observed symptoms. The output of such a system is a diagnosis (and possibly an explanation or justification) [1]. In many applications of interest, it is desirable for the system to not only identify the possible causes of the problem, but also to suggest suitable remedies (systems capable of advising) or to give a reliability rate of the identification of possible causes. Recently, several decision support systems and intelligent systems have been developed [2,3] and the diagnosis approaches based on such intelligent systems have been developed for industrial applications $[1,4,5]$, and biomedicine applications [6-10]. Currently, one of the most used approaches to feature identification, classification, and 
decision-making problems inherent to fault detection and diagnosis, is soft computing implying mainly neural networks and fuzzy logic $[1,3-6,9,10]$.

Over the past decades, new approaches based on artificial neural networks have been developed aiming to solve real life problems related to optimization, modeling, decisionmaking, classification, data mining, and nonlinear functions (behavior) approximation. Inspired from biological nervous systems and brain structure, artificial neural networks could be seen as information processing systems, which allow elaboration of many original techniques covering a large application field based on their appealing properties such as learning and generalization capabilities [11-13].

Another aspect of increasing importance, and strongly linked to data processing and the amount of data available concerning processes or devices (due to the high level of sensors and monitoring), is the extraction of knowledge from data to discover the information structure hidden in it. Several approaches have been developed to analyze and classify biomedicine signals: electroencephalography signals [7], electrocardiogram signals [8], and particularly signals based on Auditory Brainstem Response (ABR) test, which is a test for hearing and brain (neurological) functioning, [6,14-16]. Traditionally, biomedicine signals are processed using signal processing approaches, mainly based on peak and wave identification from pattern recognition approaches, such as in $[6-8,14-16]$. The main problem is then to identify pertinent parameters. This task is not trivial, because the time (or frequency) is not always the variable that points up the studied phenomena's features leading then to a necessity of multiple knowledge representations (signal, image, ...).

This paper deals with pattern recognition (classification) and decision-making based on Artificial Intelligence using soft computing implying neural networks and fuzzy logic applied to a biomedicine problem. The aim of this paper is absolutely not to replace specialized human but to suggest a decision support tool with a satisfactory reliability degree for Computer-Aided Diagnosis (CAD) systems. Thus, a global diagnosis synopsis is deduced from a fault diagnosis system analysis of the classification and decision-making problem, given in Sect. 2. Afterwards, the decision-making problem from the results of two neural classifications is stated and a hybrid intelligent diagnosis approach is suggested in Sect. 3. In Sects. 4 and 5, the suggested approach is developed for a computer-aided auditory diagnosis, from signal and image representations, in order to achieve a diagnosis tool able to assert auditory pathologies based on ABR test which provides an effective measure of the integrity of the auditory pathway. Then, prototype design and experimental results are presented, and a discussion is given with regard to reliability and large application field.

\section{Fault diagnosis system analysis}

Globally, the main goals of fault diagnosis systems for CAD $[5,10]$ are: to detect if a fault is in progress as soon as possible, to classify the fault in progress, to be able to suggest suitable remedies (systems able of advising) or to give a reliability rate of the identified fault through a Confidence Index $(C I)$.

$\mathrm{CAD}$ is an attractive area leading to future promising fault diagnosis applications. However, dealing with expert (human) knowledge consideration, the computer-aided diagnosis dilemma is one of most interesting, but also one of the most difficult problems. The fault diagnosis help is often related to the classification of several information sources implying different representations. Fault diagnosis can be obtained from the classification of only one kind of information (knowledge) representation. However, experts use several information to emit their diagnosis. Then, an interesting way to built efficient fault diagnosis system can be deduced from this concept in order to take advantage from several information. More, experts can use several information sources, in various forms; qualitative or quantitative data, signals, images, to emit their diagnosis. Thus, these information could be issued from different information sources and/or from different representations of a same test. For instance, in case of diagnosis of the same fault classes set, one can consider that these information are independently, in parallel, classified and after the decision-making of their
Fig. 1 Global diagnosis synopsis of the same fault classes set

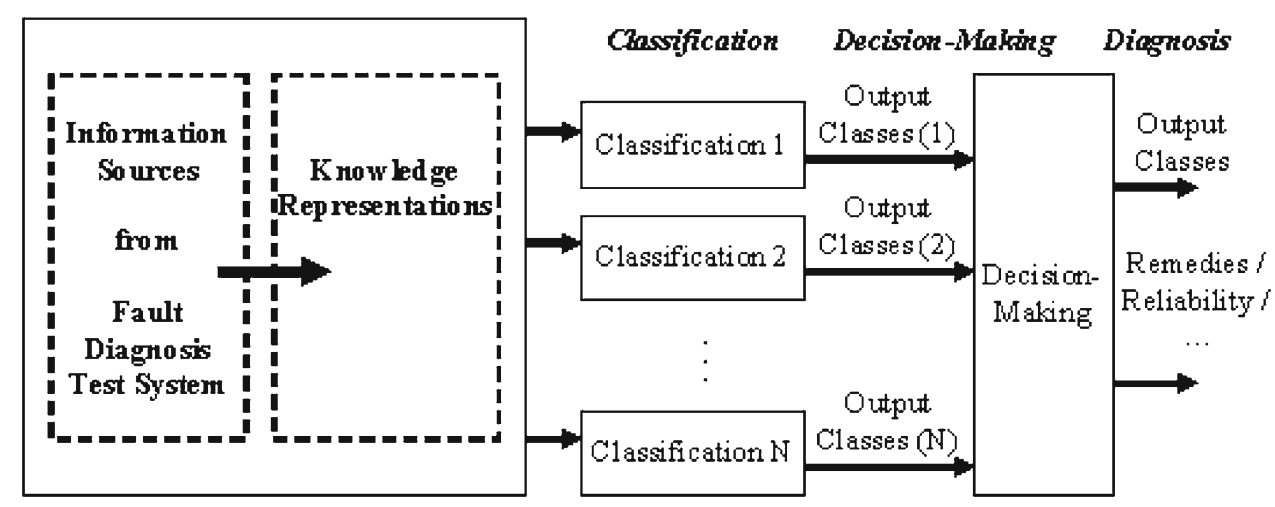


results gives then final results as shown in Fig. 1. Final results give the fault classes set and suitable remedies or a reliability rate of the possible identified fault class.

\section{Hybrid intelligent diagnosis approach}

In order to study the decision-making phase of the global diagnosis synopsis suggested in Fig. 1, two different knowledge representations are considered from only one information source, as shown in Fig. 2. This configuration, in the case of diagnosis of the same fault classes set, leads to two different classifications. More, if such classifications are handled by neural networks, which are known to be appropriate for classification $[11-13,17]$ the decision-making appears to be difficult particularly in CAD. In such cases, CAD can be useful and efficient only if the results are given with a reliability parameter (e.g., a $C I$ on each fault classes set result).

In fact, the classification stage consists of the signal classification which can be based on multilayer feedforward perceptron networks (MLP) or on radial basis function networks (RBF) networks as well as the image classification which can be based on the same networks. These networks are chosen from their theoretical and practical features particularly the fact that MLP are neural global approximators, whereas RBF are neural local approximators [11]. Practically, even if RBF classifiers usually converge faster than MLP in general during training, they are almost equivalent in terms of classification performance from a same knowledge representation of an information source. The interest here is to exploit these classifiers from two different knowledge representations of an information source. In this case, it is interesting, in a double classification, to choose (between MLP and RBF) the appropriate classifier to exploit the first knowledge representation and the appropriate one to exploit the second knowledge representation.
More, this choice is motivated by the fact that such networks can be used in a double classification in such a way to take advantage from their complementary classification performances (with a confidence parameter to enhance classification rates) as well as from their competitive classification performances (with the confidence parameter, this information will contribute to enhance, for instance, the $C I$ in final decision-making in case of common classification, or inversely in case of contradictory classification) [3]. Indeed, in a double classification from signal and global image, it is appropriated to classify the signal (sampled amplitude of signal which is more local than global) using neural local approximators (RBF), while, it is appropriated to classify the global image (area mean grey level of global image which is more global than local) using neural global approximators (MLP).

By another way, the nature of neural classification results (neural outputs) of the neural architectures are, in general, not binary values. In fact, for instance, the typical MLP or RBF used for classification with sigmoïdal outputs give output class values between $[0,1]$ or outputs which are distances from RBF centers, respectively. This makes difficult the problem of the decision-making from two neural networks.

The analysis of neural classifier outputs shows that, in case of MLP, more the output is close to 1 and more this output will be close to be the identified fault class. Contrarily, more the output is close to 0 and more this output will be far to be the identified fault class. In case of RBF, the outputs are distances from RBF centers. In this case with a new scale of outputs it is easily to make output class values varying between $[0, \mathrm{c}]$, where $\mathrm{c}$ is a constant to be determined (e.g., see Sect. 5.1, Fig. 7a-c). Then, more the output which is a distance is close to $\mathrm{c}$ and more this output will be far to be identified as fault class. Contrarily, more the output is close to 0 and more this output will be close to be the identified fault class. From this purpose, one interesting way to built
Fig. 2 Decision-making from two neural classifications (diagnosis of the same fault classes set)

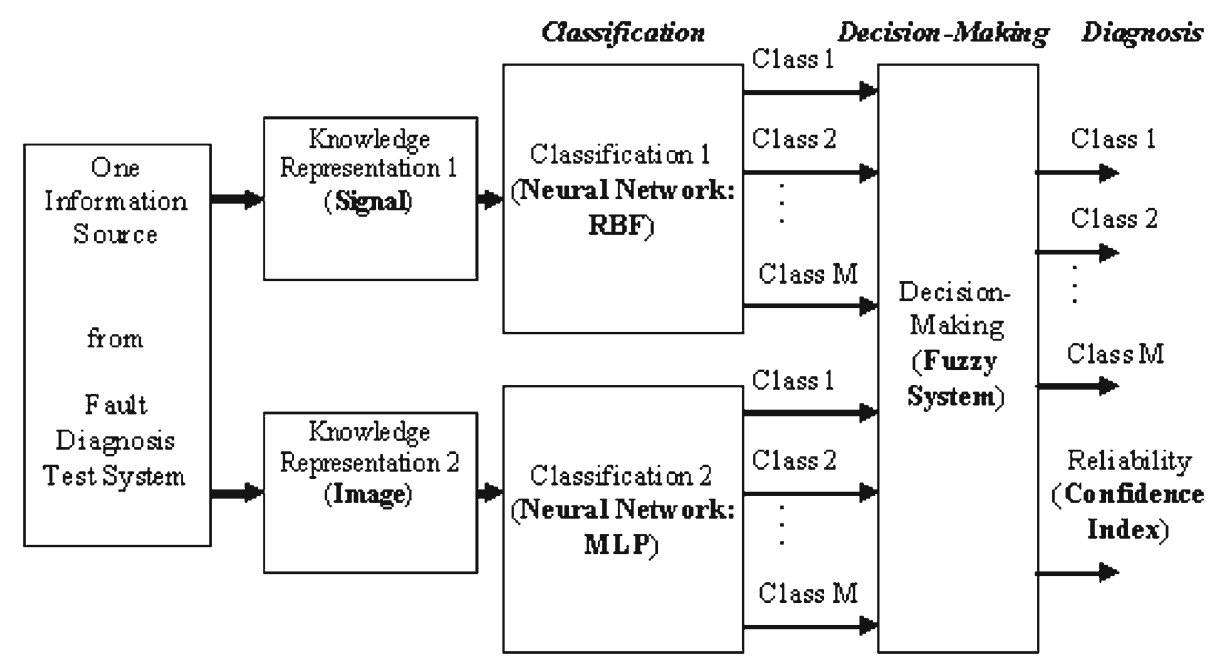


efficient decision-making from two neural classifiers is fuzzy $\operatorname{logic}[18,19]$.

Elsewhere, such decision-making system should be useful and efficient giving a reliability parameter, e.g., a Confidence Index $(\mathrm{CI})$ on each fault classes set result.

Then, a first way is to design a fuzzy system with seven (07) inputs (three inputs from first classifier, three inputs from second classifier, and one confidence parameter input) leading to a fuzzy rule base built of $3^{7}=2,187$ rules which is unfortunately a huge rule number difficult and hard to implement.

An interesting way is then to design two fuzzy classifiers for the decision-making:

- the first fuzzy system for the primary decision-making from two neural classifiers with (06) inputs (three inputs from first classifier and three inputs from second classifier) leading to a fuzzy rule base built of $3^{6}=729$ rules,

- the second fuzzy system for the final decision-making from the first fuzzy system and a confidence parameter, i.e., with four (04) inputs (three inputs from first fuzzy system and one confidence parameter input) leading to a fuzzy rule base built of $3^{4}=81$ rules.

Thus, two fuzzy decision-making systems are necessary, avoiding a decision system with a huge rule number and associating a confidence parameter to the decision, in order to decide from two neural classifiers and to give a reliability parameter (e.g., a Confidence Index CI) for a useful and efficient CAD.

Thus, the results of the two neural classifications, from knowledge representation 1 and knowledge representation 2, see Fig. 2, can be then efficiently exploited in a fuzzy system to ensure a satisfactory reliability. The fuzzy decisionmaking system based on a fuzzy inference can be exploited in order to capture the expert (human) knowledge [2,20]. Then, the decision-making system allows to decide the fault classes diagnosis among: Class 1, Class 2,..., and Class $\mathrm{M}$, and its usefulness and efficiency are better traduced with the associated $C I$ on its decision. Contrary to a time or frequency (signal) based representation, the image based one, taking benefit from it's 2-D nature, offers advantage a richer representation allowing to take into account more complex features (shapes, particular information, ...).

\section{Biomedical application: computer-aided auditory diagnosis}

The ABR test involves attaching electrodes to the head to record electrical activity from the auditory nerve (the hearing nerve) and other parts of the brain. This recorded electrical activity is known as Brainstem Auditory Evoked Potentials (BAEP).

\subsection{Brainstem auditory evoked potentials (BAEP) clinical test}

When a sense organ is stimulated, it generates a string of complex neurophysiology processes. BAEP are electrical response caused by the brief stimulation of a sense system. The stimulus gives rise to the start of a string of action's potentials that can be recorded on the nerve's course, or from a distance of the activated structures. BAEP are generated as follows (see Fig. 3a): the patient hears clicking noise or tone bursts through earphones. The use of auditory stimuli evokes an electrical response. In fact, the stimulus triggers a number of neurophysiology responses along the auditory pathway. An action potential is conducted along the eight nerve, the brainstem, and finally to the brain. A few times after the initial stimulation, the signal evokes a response in the area of brain where sounds are interpreted.

\subsection{Extraction of the two knowledge representations (signal and image)}

A technique of extraction [15] allows us, following 800 acquisitions such as described before, the visualization of the BAEP estimation on averages of 16 acquisitions. Thus, a surface of 50 estimations called Temporal Dynamic of the Cerebral trunk (TDC) can be visualized. The average signal, which corresponds to the average of the 800 acquisitions, and the TDC surface could then be obtained. Those are then processed into a signal representation as shown in Fig. 3 b. In this figure, an example of TDC surface for a patient is shown. The average signal (named signal representation) is presented in front of TDC surface which is better shown in Fig. 3c.

Three patient classes are studied: Retro-cochlear auditory disorder patients (Retro-cochlear Class: RC), Endocochlear auditory disorder patients (Endo-cochlear Class: EC), healthy patients (Normal Class: NC). Figure 4a-c shows examples of signal representations for two patients: RC, EC, and $\mathrm{NC}$, respectively.

The signal to image conversion (named image representation), shown in Fig. 5, is obtained after a TDC surface signal and image processing [9,21]. Figure 5 presents image representations for the same six patients.

These figures (Figs. 4, 5) illustrate the fact that, signal or image representations could be very similar for patients belonging to different classes, and they could be very different for patients belonging to a same class, demonstrating the difficulty of their classification. 
Fig. 3 a BAEP clinical test. b TDC surface. c Average signal processing (b)

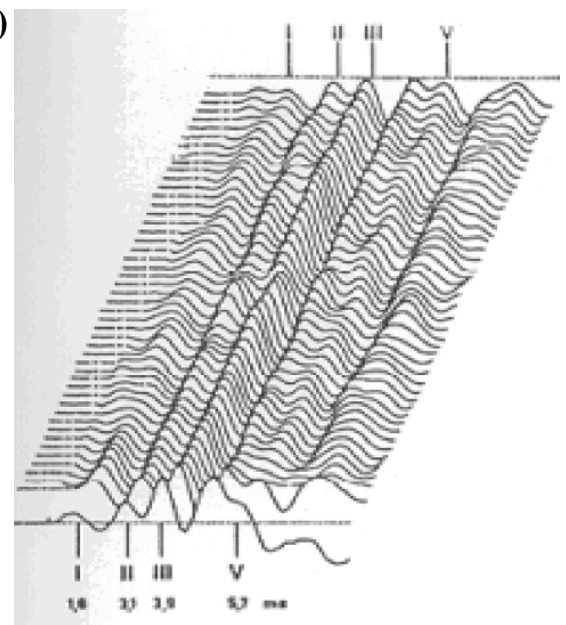

(c)

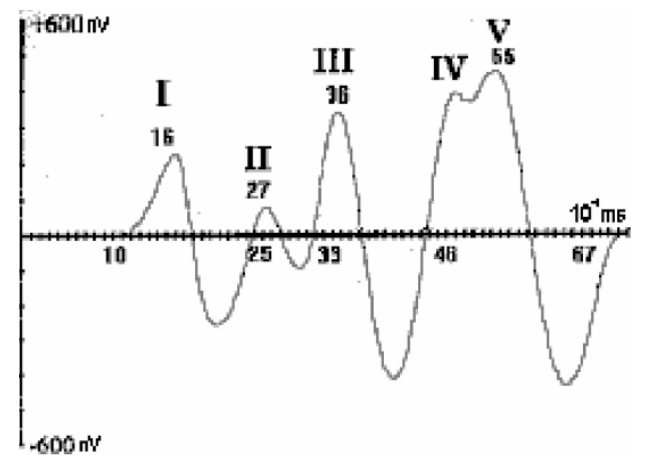

\subsection{Suggested hybrid intelligent diagnosis system}

The hybrid intelligent diagnosis system suggested in Fig. 6 is built of data processing stage, classification stage, primary fuzzy decision-making stage leading to a primary diagnosis, and final fuzzy decision-making stage leading to the final diagnosis. Note that this suggested diagnosis system is deduced from the synopsis of classification and decisionmaking presented in Fig. 2.

The data processing stage consists of extracting signal and image representations from data source (signals: TDC surface) and deducing the signal data and image data.

The classification stage consists of the signal classification which is based on RBF networks while the image classification is based on MLP networks. This choice is mainly based on the two facts (discussed in Sect. 3) that:

- MLP and RBF networks can be used in a double classification in such a way to take advantage from their complementary classification performances (with a confidence parameter to enhance classification rates) as well as from their competitive classification performances [3],

- MLP are neural global approximators, whereas RBF are neural local approximators [11].
The primary and final fuzzy decision-making stages consist of the Primary Fuzzy System (PFS) and Final Fuzzy System (FFS), respectively. These fuzzy decision-making systems are used to capture the decision-making behavior of a human expert while giving the appropriate diagnosis $[2,17]$, i.e., it must mimic the input/output mapping of this human expert. Note that the two fuzzy inferences of PFS and FFS, based on Mamdani's fuzzy inference, are developed as detailed in the diagnosis approach using only image representation described in [9] with the simplification detailed in [22]. From this simplification, the fuzzy rule base of PFS which is built of $3^{6}=729$ rules will make in use only $2^{6}=$ 64 rules in each inference, while the fuzzy rule base of FFS which is built of $3^{4}=81$ rules will make in use only $2^{4}=16$ rules in each inference.

Thus, the double classification, from signal representation and image representation, is exploited in PFS to ensure a satisfactory reliability for a computer-aided auditory diagnosis. Input parameters, obtained from the two neural networks, of PFS are RC_S $, E C \_S, N C \_S, R C_{-} I, E C_{-} I$, and $N C_{-} I$. Thus, for each input, $\mathrm{PFS}$ is able to decide of appropriate diagnosis among Primary Diagnosis outputs $P D_{R C}, P D_{E C}$, and $P D_{N C}$.

The diagnosis reliability obtained from the PFS is reinforced (enhanced) using the obtained diagnosis result associated with a confidence parameter, Auditory Threshold $(A T)$ of 

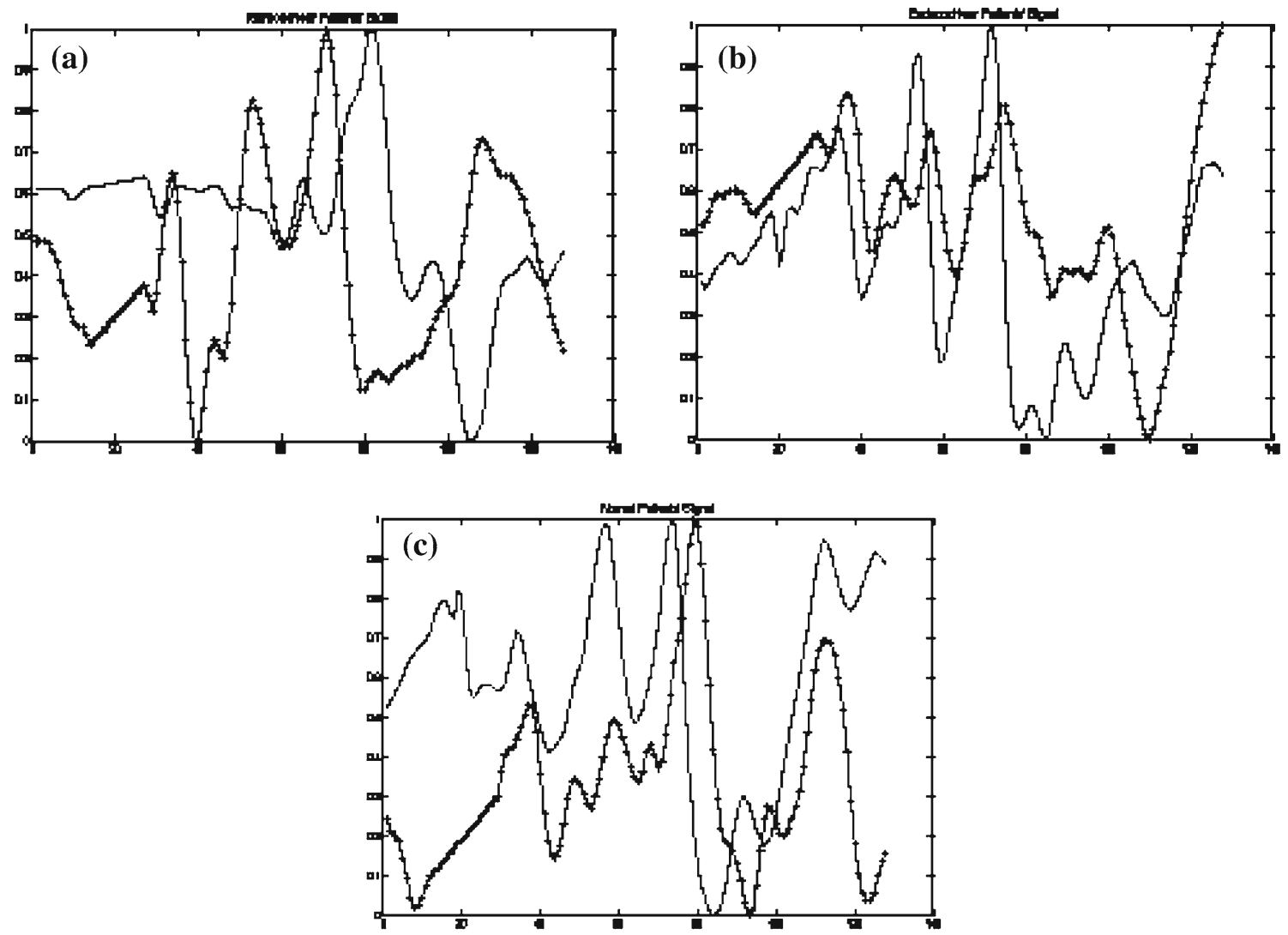

Fig. 4 Two examples of signal representations for patients. a RC. b EC. c NC

(a)

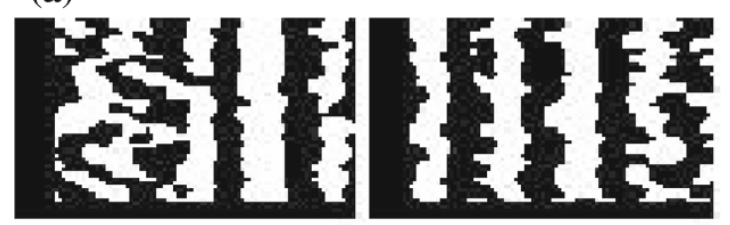

(b)

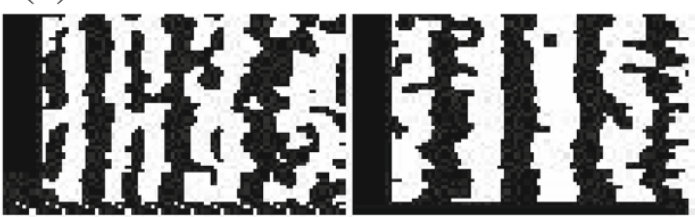

(c)
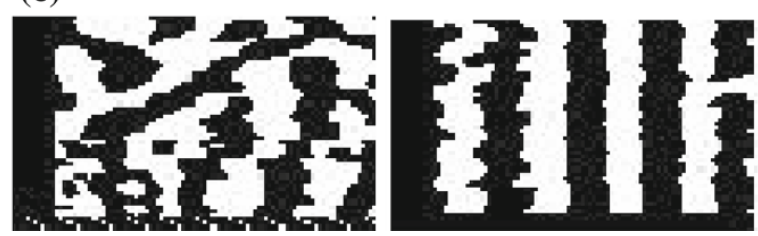

Fig. 5 Two examples of image representations for patients. a RC. b EC. c NC

patients, used as a confidence parameter, exploited in FFS in order to generate the final diagnosis result. Input parameters, issued from PFS, of FFS are $A T, P D_{R C}, P D_{E C}$, and $P D_{N C}$. Thus, for each input, FFS is able to decide of the appropriate diagnosis among Final Diagnosis outputs: $F D_{R C}, F D_{E C}$, and $F D_{N C}$ with their Confidence Index $(C I)$.

\section{Prototype design and experimental results}

For the validation of the suggested intelligent system, in the case of auditory diagnosis help, the used data base is issued from a specialized center in functional explorations in otoneurology CEFON ("Centre d'Explorations Fonctionnelles Oto-Neurologiques, Paris, France.”) [15]. This knowledge base is depicted for learning and for generalization in Table 1.

\subsection{Prototype design}

The neural classification results are presented in Table 2 for signal classification and in Table 3 for image classification. Learning database has successfully been learnt by the two classifications. The global correct classification rate is quite 
Fig. 6 Hybrid intelligent diagnosis system synopsis for auditory diagnosis help. $R B F$ radial basis function network, $R C$ Retro-cochlear Class, $M L P$ multilayer perceptron network, $E C$ Endo-cochlear class, $S$ signal, $I$ image, $N C$

normal-cochlear class, $A T$ auditory threshold, $P D$ primary diagnosis outputs, $F D$ final diagnosis outputs, $C I$ confidence index

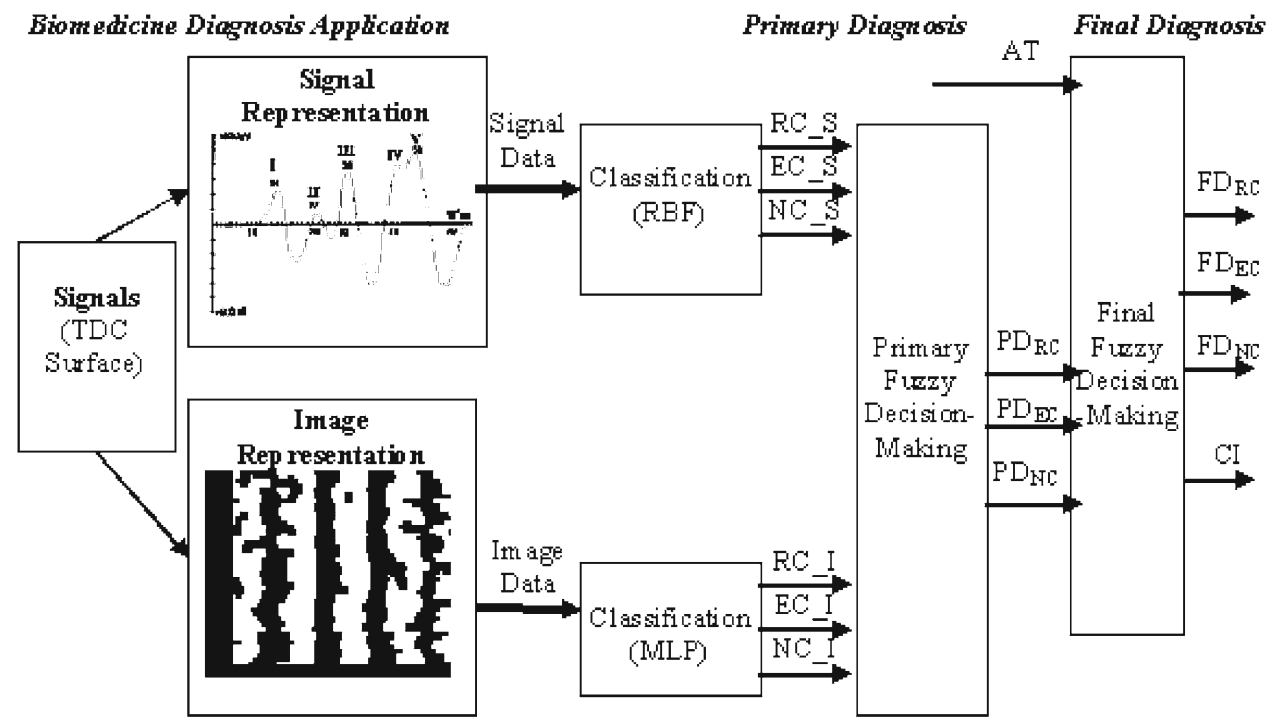

Table 1 Signal neural classification results (RBF)

\begin{tabular}{lll}
\hline Knowledge base & $\begin{array}{l}\text { Learning } \\
\text { base }\end{array}$ & $\begin{array}{l}\text { Generalization } \\
\text { base }\end{array}$ \\
\hline Retro-cochlear & 11 & 27 \\
Endo-cochlear & 6 & 71 \\
Normal & 7 & 84 \\
\hline
\end{tabular}

Table 2 Signal neural classification results (RBF)

\begin{tabular}{lll}
\hline Signal results (RBF) & $\begin{array}{l}\text { Learning } \\
\text { rate }(\%)\end{array}$ & $\begin{array}{l}\text { Generalization } \\
\text { rate }(\%)\end{array}$ \\
\hline Retro-cochlear & 100 & 44.44 \\
Endo-cochlear & 100 & 52.11 \\
Normal & 100 & 58.33 \\
\hline
\end{tabular}

Table 3 Image neural classification results (MLP)

\begin{tabular}{lll}
\hline Image results (MLP) & $\begin{array}{l}\text { Learning } \\
\text { rate }(\%)\end{array}$ & $\begin{array}{l}\text { Generalization } \\
\text { rate }(\%)\end{array}$ \\
\hline Retro-cochlear & 100 & 29.62 \\
Endo-cochlear & 100 & 35.21 \\
Normal & 100 & 70.23 \\
\hline
\end{tabular}

similar for the two classifications, $51.62 \%$ for signal one and $45.02 \%$ for image one. However, correct classification rate is more homogeneous in case of signal classification. Image classification allows to obtain as far as $70.23 \%$ of correct classification for NC. Obtained rates for RC and EC are then low and quite similar. With the two classifications, if EC are incorrectly classified by neural classifications, obtained class is NC (in majority). And, if NC are incorrectly clas- sified by neural classifications, the obtained class is EC (in majority).

Primary Fuzzy System (PFS) The double classification, from signal representation and image representation is exploited in a Primary Fuzzy System (PFS) to ensure a satisfactory reliability of a Primary Diagnosis (PD). In order to exploit the expert (human) knowledge [2], the fuzzy decisionmaking system, developed in this Section, based on Mamdani's fuzzy inference must be able to decide of the appropriate PD among RC $\left(P D_{R C}\right)$, EC $\left(P D_{E C}\right)$, and Normal Class $\left(P D_{N C}\right)$. The fuzzy decision-making system is suggested to the diagnosis decision-making help, i.e., to select the appropriate primary diagnosis for each patient among $P D_{R C}, P D_{E C}$, and $P D_{N C}$.

The input parameters are $R C \_S, E C \_S, N C \_S, R C \_I, E C \_I$, and $N C_{-} I$. These inputs are obtained from neural networks, i.e., scaled from 0 to 1 .

Then, the membership functions of $R C_{-} S, E C_{-} S$, and $N C \_S$ are defined as shown in Fig. 7a-c, where Near $(\mathrm{N})$, Medium (M), and Far (F) are the fuzzy variables. The membership functions of $R_{-} C_{-} I, E C_{-} I$, and $N_{-} I$ are defined as shown in Fig. 7d-f, where Far (F), Medium (M), and Near $(\mathrm{N})$ are the fuzzy variables.

Thus, the input vector is then the vector $\mathbf{I}=\left[R_{C} S\right.$, $\left.E C \_S, N C \_S, R C \_I, E C \_I, N C \_I\right]$. For each input, this fuzzy decision-making system must be able to select the appropriate primary diagnosis.

The fuzzy decision-making system is used to capture the decision-making behavior of a human expert while giving the appropriate diagnosis [17], i.e., it must mimic the input/output mapping of this human expert. Indeed, the latter has formulated his knowledge in a linguistic form which provides an explanation to give an appropriate primary diagnosis. 
(a)

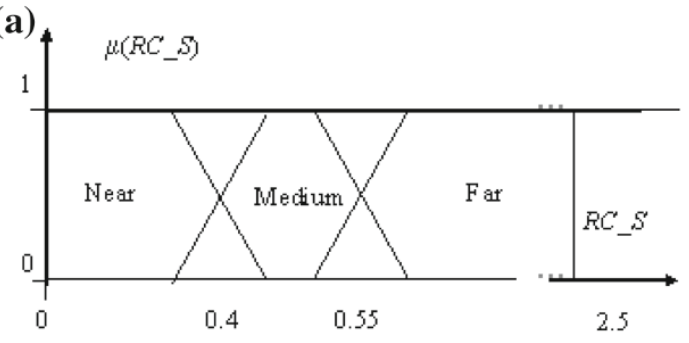

(c)

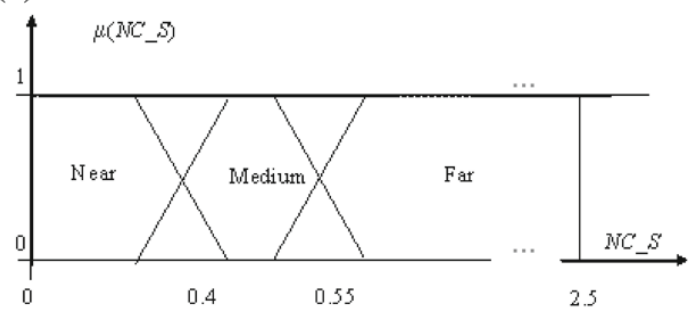

(e)

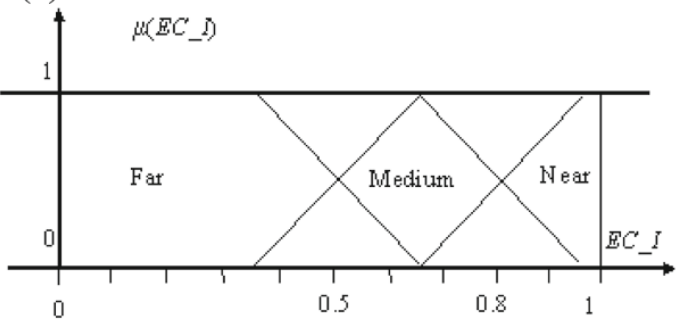

(b)

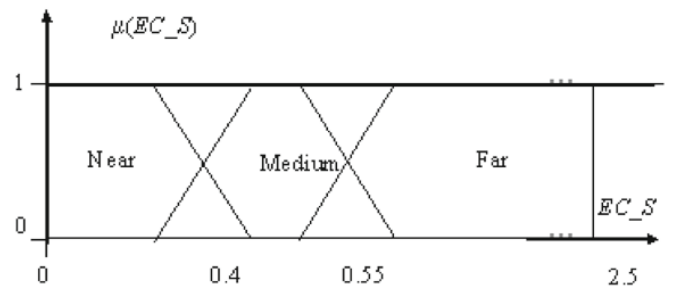

(d)

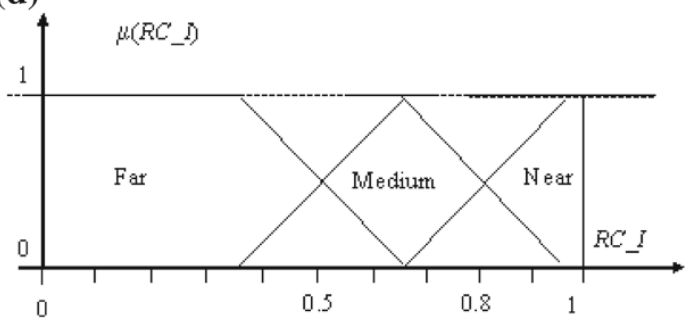

(f)

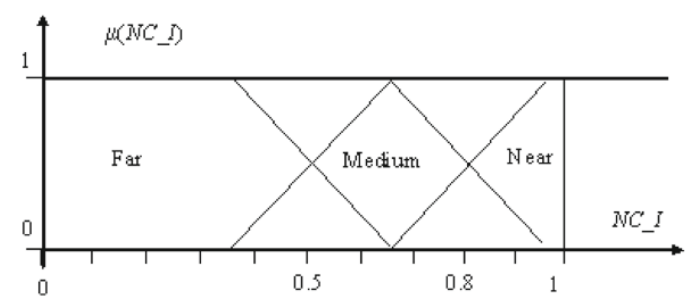

Fig. 7 Membership functions of: a $R C_{-} S$. b $E C \_S$. c $N C \_S$. d $R C_{-} I$. e $E C_{-} I$. f $N C_{-} I$

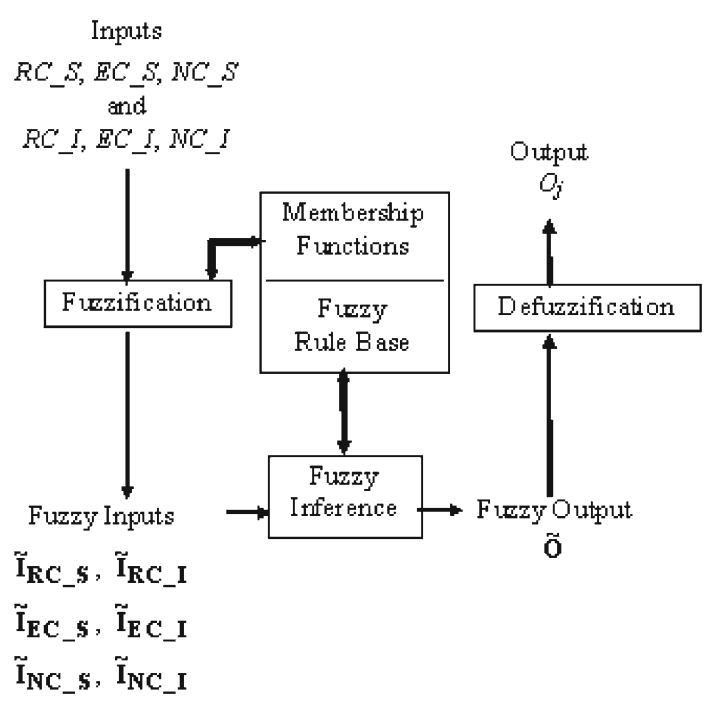

Fig. 8 Fuzzy decision-making system: Primary Fuzzy System (PFS)

To mimic this diagnosis, the fuzzy linguistic formulation is used and a set of fuzzy rules are then established. Thus, these fuzzy rules are used to incorporate this human expert knowledge in the suggested fuzzy decision-making system, illustrated in Fig. 8, where the vectors $\tilde{\mathbf{I}}_{\mathbf{R C} \_\mathbf{S}}, \tilde{\mathbf{I}}_{\mathbf{E C} \_}$s, $\tilde{\mathbf{I}}_{\mathbf{N C} \_\mathbf{S}}, \tilde{\mathbf{I}}_{\mathbf{R C} \_\mathbf{I}}$,
$\tilde{\mathbf{I}}_{\mathbf{E C} \_\mathbf{I}}$, and $\tilde{\mathbf{I}}_{\mathbf{N C} \_\mathbf{I}}$ represent the fuzzy vectors of the input components $R C \_S, E C \_S, N C \_S, R C \_I, E C \_I$, and $N C \_I$, respectively; while $\tilde{\mathbf{O}}$ represent the fuzzy vector of the output $O j$ which is a component of the vector $\mathbf{O}=\left[P D_{R C}, P D_{E C}\right.$, $\left.P D_{N C}\right]$ where $P D_{R C}, P D_{E C}, P D_{N C}$ are Primary Diagnosis outputs RC, EC, and Normal Class, respectively.

The operation of the fuzzification calculates the degrees for each evaluated parameter (input) belonging to the three membership functions, e.g., for $R C \_S$ this operation calculates $\left\{\mu_{N}\left(R C \_S\right), \mu_{M}\left(R C \_S\right), \mu_{F}\left(R C \_S\right),\right\}$ with $\mu_{N}\left(R C \_S\right)$, $\mu_{M}\left(R C \_S\right)$ and $\mu_{F}\left(R C \_S\right)$ the membership degrees of fuzzy sets $\mathrm{N}, \mathrm{M}$, and $\mathrm{F}$, respectively.

The fuzzy rule base is built of $3^{6}=729$ rules deduced from the six (06) inputs where each input has three (03) fuzzy variables. Thus, established fuzzy rules are:

If $\left(R C_{-} S\right.$ is $\mathrm{N}$ and $E C_{-} S$ is $\mathrm{N}$ and $N_{-} C_{-} S$ is $\mathrm{N}$ and $R C_{-} I$ is $\mathrm{F}$ and $E C_{-} I$ is $\mathrm{F}$ and $N C_{-} I$ is $\mathrm{F}$ ) Then $\tilde{\mathbf{O}}=\left[\mu\left(\mathrm{PD}_{\mathrm{RC}}\right), \mu\left(\mathrm{PD}_{\mathrm{EC}}\right), \mu\left(\mathrm{PD}_{\mathrm{NC}}\right)\right]$, If $\left(R C_{-} S\right.$ is $\mathrm{N}$ and $E C_{-} S$ is $\mathrm{N}$ and $N_{C} C_{-} S$ is $\mathrm{N}$ and $R C_{-} I$ is $\mathrm{F}$ and $E C_{-} I$ is $\mathrm{F}$ and $N C_{-} I$ is $\left.\mathrm{M}\right)$ Then $\tilde{\mathbf{O}}=\left[\mu\left(P D_{R C}\right), \mu\left(P D_{E C}\right), \mu\left(P D_{N C}\right)\right]$, ... If $\left(R C_{-} S\right.$ is $\mathrm{F}$ and $E C_{-} S$ is $\mathrm{F}$ and $N C_{-} S$ is $\mathrm{F}$ and $R C_{-} I$ is $\mathrm{N}$ and $E C_{-} I$ is $\mathrm{N}$ and $N C_{-} I$ is $\mathrm{M}$ ) Then 
$\tilde{\mathbf{O}}=\left[\mu\left(P D_{R C}\right), \mu\left(P D_{E C}\right), \mu\left(P D_{N C}\right)\right]$,

If $\left(R C_{-} S\right.$ is $\mathrm{F}$ and $E C_{-} S$ is $\mathrm{F}$ and $N C_{-} S$ is $\mathrm{F}$ and $R C_{-} I$

is $\mathrm{N}$ and $E C_{-} I$ is $\mathrm{N}$ and $N C_{-} I$ is $\mathrm{N}$ ) Then

$\tilde{\mathbf{O}}=\left[\mu\left(P D_{R C}\right), \mu\left(P D_{E C}\right), \mu\left(P D_{N C}\right)\right]$.

In this fuzzy rule base, the fuzzy decision-making vector $\tilde{\mathbf{O}}$ is expressed by:

\section{õ}

$=\left[\mu_{\left(\mathrm{RC} \_S m\right.}, \mathrm{EC} \_\mathrm{Sm}, \mathrm{NC} \_\mathrm{Sm}, \mathrm{RC} \_\mathrm{Im}, \mathrm{EC} \_\mathrm{Im}, \mathrm{NC} \_\mathrm{Im}\right)\left(P D_{R C}\right)$,

$\mu$ (RC_Sm,EC_Sm,NC_Sm, RC_Im, EC_Im,NC_Im) $\left(P D_{E C}\right)$,

$\left.\left.\mu_{\left(\mathrm{RC} \_S m\right.}, \mathrm{EC} \_\mathrm{Sm}, \mathrm{NC} \_\mathrm{Sm}, \mathrm{RC} \_\mathrm{Im}, \mathrm{EC} \_\mathrm{Im}, \mathrm{NC} \_\mathrm{Im}\right)\left(P D_{N C}\right)\right]$

where $\mu$ (RC_Sm,EC_Sm,NC_Sm,RC_Im,EC_Im,NC_Im) $\left(O_{j}\right)$ represents the membership function degree of $O_{j}$ with $m=1$ or 2 , see the simplification given below.

The fuzzy inference is achieved by the Min and Max operations. The particularity of the input parameters is that, for each given input, at least one membership function degree (among the three membership function degrees) is always equal to zero. Consequently, only two (02) membership function degrees are to be considered [22]. From this simplification, for each specific decision-making situation, the values of inputs are mapped to the discrete intervals to form the fuzzy sets:

$\tilde{\mathbf{I}}_{\mathbf{R C} \_\mathbf{S}}=\left\{\mu_{1}\left(R C_{-} S\right), \mu_{2}\left(R C_{-} S\right)\right\}$,

$\tilde{\mathbf{I}}_{\mathbf{E C} \_\mathbf{S}}=\left\{\mu_{1}\left(E C_{-} S\right), \mu_{2}\left(E C_{-} S\right)\right\}$,

$\tilde{\mathbf{I}}_{\mathbf{N C} \_} \mathbf{S}=\left\{\mu_{1}\left(N C_{-} S\right), \mu_{2}\left(N C_{-} S\right)\right\}$,

$\tilde{\mathbf{I}}_{\mathbf{R C} \_\mathbf{I}}=\left\{\mu_{1}\left(R C_{-} I\right), \mu_{2}\left(R C_{-} I\right)\right\}$,

$\tilde{\mathbf{I}}_{\mathbf{E C} \_\mathbf{I}}=\left\{\mu_{1}\left(E C_{-} I\right), \mu_{2}\left(E C_{-} I\right)\right\}$,

$\tilde{\mathbf{I}}_{\mathbf{N C} \_\mathbf{I}}=\left\{\mu_{1}\left(N C_{-} I\right), \mu_{2}\left(N C_{-} I\right)\right\}$,

where for instance $\mu_{\mathrm{m}}\left(R C_{-} S\right)$, with $\mathrm{m}=1$ or 2 , are the membership function degrees of the input $R C_{-} S$. With this description, one can have $2^{6}=64$ possible conditions corresponding to sixty four (64) fuzzy rules. Then, the level of certainty of each condition $\mu_{1}, \mu_{2}, \ldots, \mu_{64}$ can be found using the Min operation:

$$
\begin{aligned}
& \mu_{\text {cond }}(\text { RC_S1, EC_S1, NC_S1, RC_I1, EC_I1, NC_I1) } \\
& =\operatorname{MIN}\left(\mu_{1}\left(R C \_S\right), \mu_{1}\left(E C \_S\right), \mu_{1}\left(N C \_S\right), \mu_{1}\left(R C_{-} I\right)\right. \text {, } \\
& \left.\mu_{1}\left(E C_{-} I\right), \mu_{1}\left(N C_{-} I\right)\right)=\mu_{1} \text {, } \\
& \mu_{\text {cond }}(\text { RC_S1, EC_S1, NC_S1, RC_I1, EC_I1, NC_I2) } \\
& =\operatorname{MIN}\left(\mu_{1}\left(R C_{-} S\right), \mu_{1}\left(E C_{-} S\right), \mu_{1}\left(N C_{-} S\right), \mu_{1}\left(R C_{-} I\right),\right. \\
& \left.\mu_{1}\left(E C_{-} I\right), \mu_{2}\left(N C_{-} I\right)\right)=\mu_{2} \text {, } \\
& \text {... } \\
& \mu_{\text {cond }}\left(R C \_S 2,\right. \text { EC_S2, NC_S2, RC_I2, EC_I2, NC_I2) } \\
& =\operatorname{MIN}\left(\mu_{2}\left(R C_{-} S\right), \mu_{2}\left(E C_{-} S\right), \mu_{2}\left(N C_{-} S\right), \mu_{2}\left(R C_{-} I\right)\right. \text {, } \\
& \left.\mu_{2}\left(E C_{-} I\right), \mu_{2}\left(N C_{-} I\right)\right)=\mu_{64} \text {, }
\end{aligned}
$$

where cond represents the fuzzy set of conditions which is written as follows: cond $=\left\{\mu_{1}, \mu_{2}, \ldots, \mu_{64}\right\}$

Each possible condition is associated with a decision-making situation $O_{j}$. Then, certainty of each situation is obtained by Max and Min operations as follows:

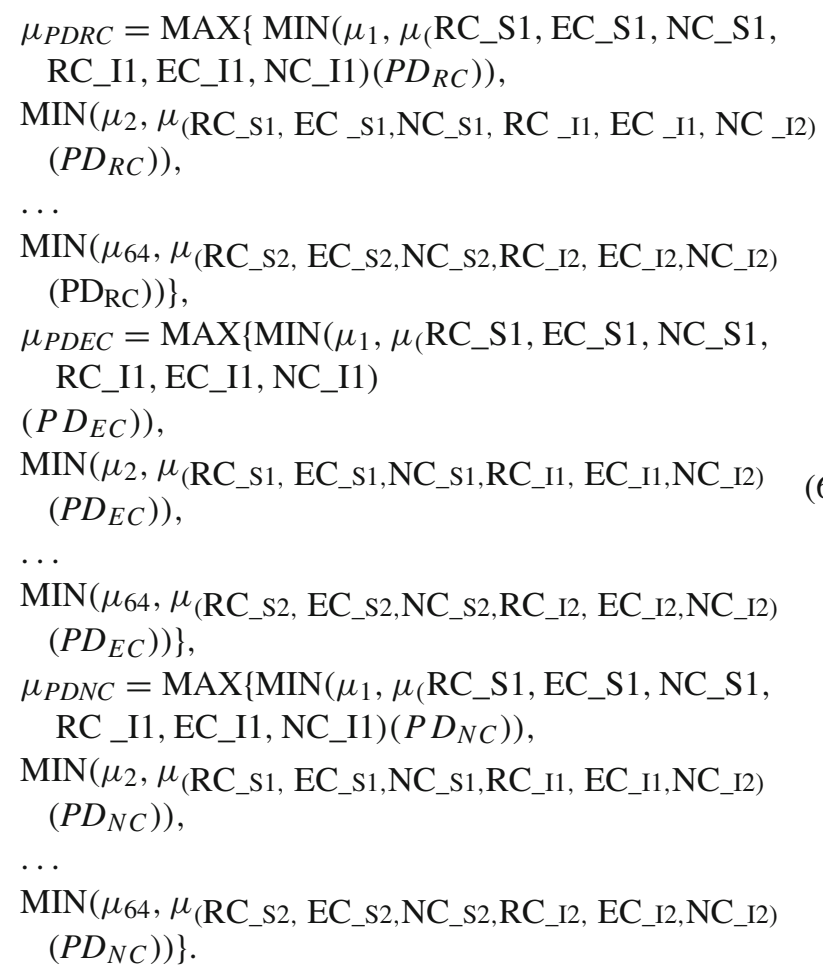

Collection of situations forms final fuzzy decision-making situation vector $\tilde{\mathbf{O}}$ :

$\tilde{\mathbf{O}}=\left\{\mu_{P D R C}\left(P D_{R C}\right), \mu_{P D E C}\left(P D_{E C}\right), \mu_{P D N C}\left(P D_{N C}\right)\right\}$

The Max operation is used for the defuzzification process to give the final decision-making situation $O_{j}$ :

$O_{j}=\operatorname{Max}\left\{\mu_{P D R C}, \mu_{P D E C}, \mu_{P D N C}\right\}$.

Final Fuzzy System (FFS) The diagnosis reliability obtained from the Primary Fuzzy System (PFS) is reinforced (enhanced) using the obtained diagnosis result with an auditory threshold parameter of patients exploited in a Final Fuzzy System (FFS) in order to generate the decision-making of the final diagnosis result. This FFS is designed and developed on the same methodology described before for the PFS, where the input parameters the Auditory Threshold (AT), $P D_{R C}, P D_{E C}$, and $P D_{N C}$ and their related membership functions are illustrated in Fig. 9a-d, respectively. The outputs of this FFS are then the outputs $F D_{R C}, F D_{E C}$, and $F D_{N C}$ giving the final diagnosis with a Confidence Index $(C I)$ of this final diagnosis. 

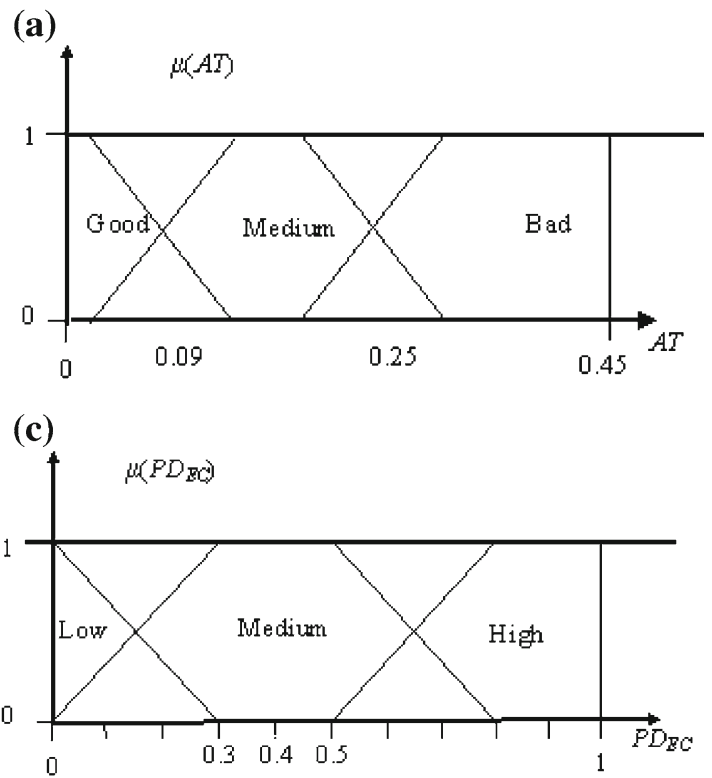

(b)

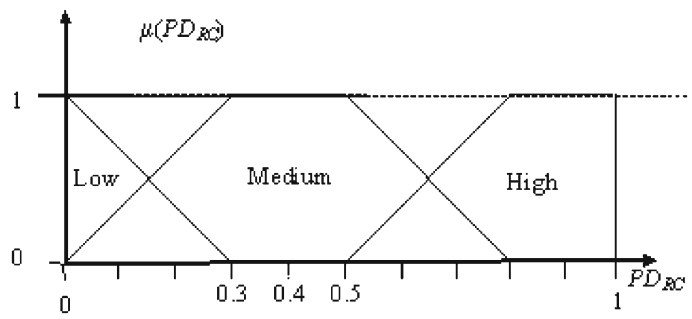

(d)

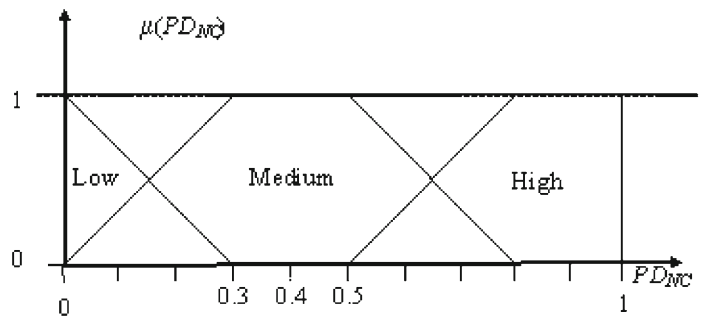

Fig. 9 Membership functions of: a auditory threshold (AT). b $P D_{R C}$. c $P D_{E C}$. d $P D_{N C}$

Table 4 Fuzzy decision-making system PFS results

\begin{tabular}{lll}
\hline $\begin{array}{l}\text { Primary fuzzy system } \\
\text { (PFS) results }(\%)\end{array}$ & $\begin{array}{l}\text { Learning } \\
\text { rate }(\%)\end{array}$ & $\begin{array}{l}\text { Generalization } \\
\text { rate }(\%)\end{array}$ \\
\hline Retro-cochlear & 100 & $33.33(37.03)$ \\
Endo-cochlear & 100 & $29.57(42.25)$ \\
Normal & 100 & $63.09(77.38)$ \\
\hline
\end{tabular}

Table 5 Fuzzy decision-making system FFS results

\begin{tabular}{lll}
\hline $\begin{array}{l}\text { Final fuzzy system } \\
(\text { FFS) results }(\%)\end{array}$ & $\begin{array}{l}\text { Learning } \\
\text { rate }(\%)\end{array}$ & $\begin{array}{l}\text { Generalization } \\
\text { rate }(\%)\end{array}$ \\
\hline Retro-cochlear & 100 & $40.74(51.85)$ \\
Endo-cochlear & 100 & $49.29(59.15)$ \\
Normal & 100 & $84.52(89.28)$ \\
\hline
\end{tabular}

\subsection{Auditory diagnosis results}

Tables 4 and 5 present the results obtained by fuzzy decisionmaking systems PFS and FFS, respectively. Rates written between brackets (x \%) represent the generalization rates calculated taking into account the patients classified simultaneously in two classes. In majority of cases, these simultaneous classifications are obtained for EC and NC. For all the classes, the generalization rate of FFS is higher than this of PFS, showing the pertinent rule of the auditory threshold. The generalization rate of normal class is clearly higher for fuzzy decision-making system FFS than for the two classifications, achieving a value of $84.52 \%(89.28 \%)$. The obtained rates for RC and EC are higher than these obtained by the image classification but quite similar than those obtained by the signal classification.
An important contribution of FFS is that it gives each fault diagnosis associated with a $C I$. This is illustrated through the following result example with a high $C I$ :

$$
\begin{aligned}
& \text { The fuzzy output }=\left\{\mu_{F D R C}, \mu_{F D E C}, \mu_{F D N C}, \mu_{C I}\right\} \\
& =\{0.05,0.94,0.05,0.96\} . \\
& \text { Then, defuzzified output }=\operatorname{Max}\left\{\mu_{F D R C}, \mu_{F D E C}, \mu_{F D N C}\right\}, \\
& =\operatorname{Max}\{0.05,0.94,0.05\}, \\
& =\mu_{F D E C}=0.94 .
\end{aligned}
$$

Then, the final result is, in this example, $\mu_{F D E C}$ and $\mu_{C I}$ equal to 0.94 and 0.96 , respectively. This result means that the identified fault (pathology) diagnosis (0.94) is EC with a high $C I(0.96)$.

\section{Discussion and conclusion}

In this paper, a hybrid intelligent diagnosis approach for computer-aided auditory diagnosis, based on neural classifications and fuzzy decision-making systems is suggested. In fact, the double classification is exploited in PFS, for a primary diagnosis, to ensure a satisfactory reliability. Second, this reliability is reinforced using a confidence parameter AT with the primary diagnosis result, exploited in FFS, in order to generate the final diagnosis giving the appropriate diagnosis with a $C I$. In effect, a first reliability degree of the suggested computer-aided diagnosis is obtained from the Primary Fuzzy System (PFS) exploiting the redundancy of the two neural classifiers. Then, this reliability degree is reinforced (enhanced) exploiting the obtained diagnosis result associated with a confidence parameter, Auditory Threshold $(A T)$ of patients in the application at hand, in the Final Fuzzy System (FFS) in order to generate the final diagnosis result 
(the appropriate diagnosis with a confidence index $C I$ ). Note that such a confidence index is very desirable in decision support systems (decision help systems), and particularly in biomedicine (e.g., biomedical application at hand, see the example given at the end of Sect. 5).

In fact, the aim is then to achieve an efficient and reliable CAD system for three classes: two auditory pathologies RC and EC and normal auditory NC. A signal and an image, issued from ABR test, are used as the two initial data representations. Implementation and experimental results are presented and discussed. The generalization rate of NC is clearly higher for PFS and FFS than for the two classifications. The obtained rates for $\mathrm{RC}$ and $\mathrm{EC}$ are higher than obtained by image classification but quite similar than those obtained by signal classification. An important contribution of the final fuzzy system FFS is that it gives each fault diagnosis associated with a $C I$.

The original contribution of this paper relies on the following points:

- the fact to process the same information source (signal) in two different ways through two different knowledge representations (signal and image). A manner to give two results from two different points of view (a kind of multiexpert results, such redundancy is desirable, particularly in biomedicine). This idea emerged from the fact that some biomedical experts are able to make a diagnosis from the observation of a signal or image biomedical results. More, usually in such biomedical applications, the knowledge representation signal is only used. In fact, the image representation offers benefit of a richer information representation than the signal one. Then, such combination (signal and image) approach will also take advantage from features which are unreachable from onedimensional signal. In effect, the interest from the image knowledge representation (of the signal) is that some pertinent features appear in such representation which are new, and interesting to exploit, with regard to the features which appear in the signal knowledge representation. Some of these signal and image pertinent features could be competitive, but also complementary. Then, both of them should be exploited to reinforce or in contrary decrease a certain diagnosis which will be traduced also in different degrees of the confidence index $(C I)$ which will be given with the associated final diagnosis result.

- the hybrid intelligent nature of the suggested diagnosis approach using an appropriate neural classification for each knowledge representation (radial basis functions RBF for signal and multilayer perceptron MLP for image), and the fuzzy system for the decision-making process from the primary diagnosis results of the two neural networks (MLP and RBF) in order to give the final diagnosis result associated with a confidence index $(C I)$.

The choice of modular neural networks have been motivated from the theoretical and practical features such as their learning and generalization capabilities as classifiers, and particularly the fact that MLP are neural global approximators (sampled amplitude of signal which is more local than global), whereas RBF are neural local approximators (area mean grey level of global image which is more global than local). More, this choice is motivated by the fact that such networks can be used in a double classification in such a way to take advantage from their complementary classification performances (with a confidence parameter to enhance classification rates) as well as from their competitive classification performances (with the confidence parameter, this information will contribute to enhance, for instance, the $C I$ in final decision-making in case of common classification, or inversely in case of contradictory classification). Due to the nature of the outputs of such neural networks (detailed in Sect. 3), one interesting way to built efficient decisionmaking from two neural networks is the choice of fuzzy logic.

Another important point motivating this choices (modular neural networks and fuzzy logic) is related to the number of fault classes used in each neural network outputs. In this paper, this number is related to the application at hand e.g., about three fault classes (Retro-cochlear Class RC, Endo-cochlear Class EC, and Normal auditory Class NC). Of course, for other applications of the suggested hybrid intelligent diagnosis this number could be more than three, in such case, the use of modular neural networks is judicious because one can always have more networks with always the number of their outputs which will be equal or less than three outputs, avoiding any combinatory explosion of the resulting number of fuzzy rules for the decision-making.

Thus, the suggested approach could be generalized to many output classes exploiting the concept of modular neural networks [3]. Such concept allows to avoid to deal with a huge number of fuzzy rules in case of a great number of output classes.

Of course, a number of current system's aspects could be investigated in order to enhance the final results. For this purpose, a fine tuning of fuzzy rules is necessary as well as a more detailed presentation of the results (the results presented are those only with a high $C I$ ).

An interesting alternative for future works, is also to investigate from rational analysis of fault diagnosis cognitive process other concepts besides fuzziness, such as causality,...

Finally, this approach could be applied to other diagnosis problem in biomedicine, where signal and image representation could be extracted from clinical tests. It is pertinent to notice that a large number of signal issued representations 
(a)

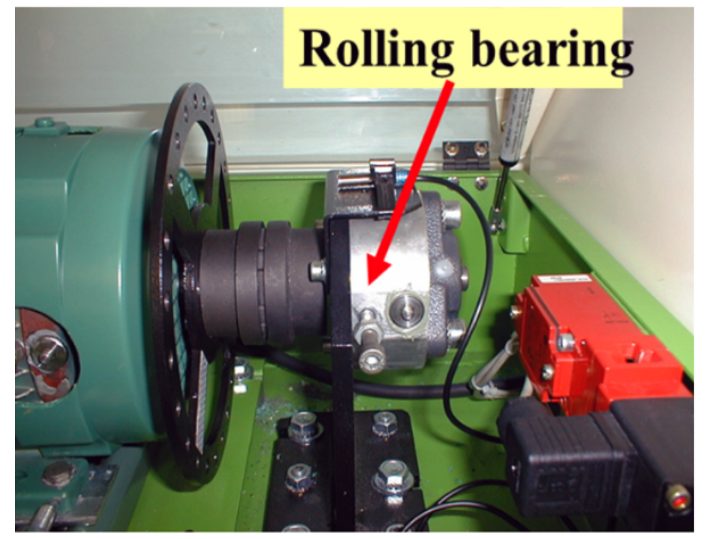

(c)
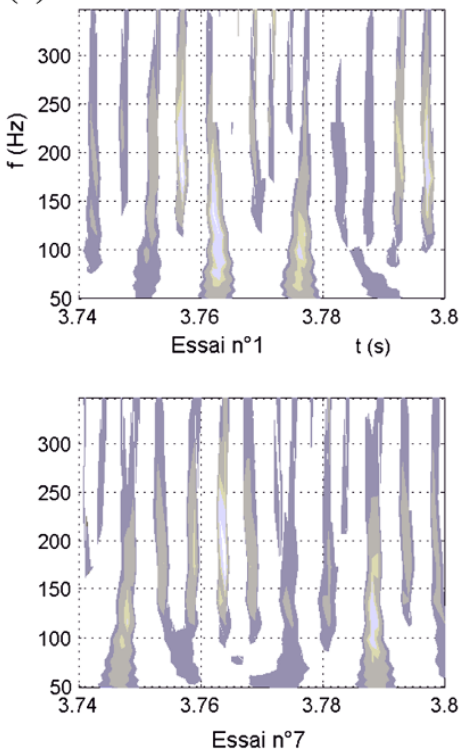

(b)
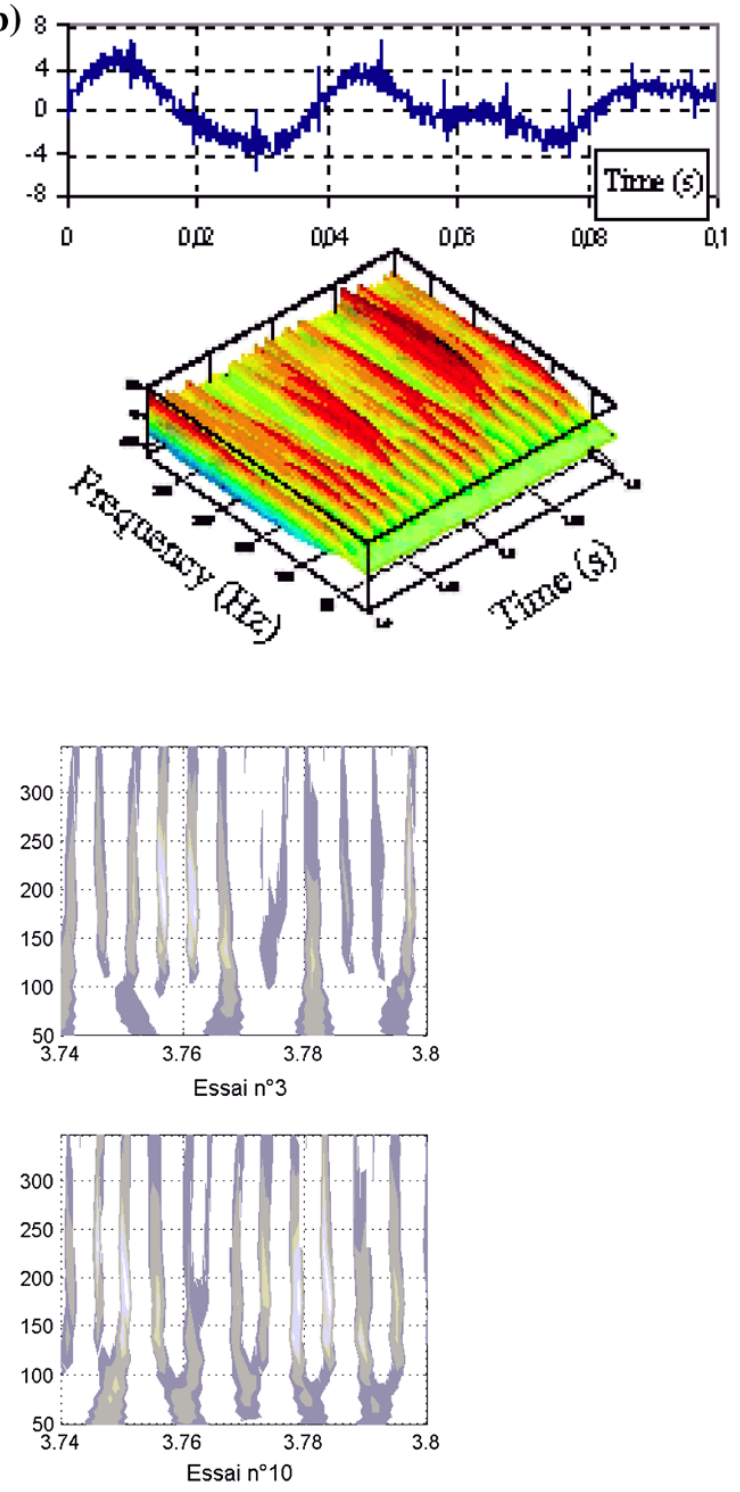

Fig. 10 Example of industrial diagnosis system and signal and image knowledge representations: a Revolving machine. b Signal knowledge representation and a wavelet transform representation. $\mathbf{c}$ Image knowledge representations (deduced from wavelet transform representation)

could be converted in image representations. Elsewhere, it could be used for industrial domain, e.g., mechatronic system as illustrated in Fig. 10, where a revolving machine is presented in Fig. 10a and two information (knowledge) representations are shown: a signal knowledge representation and a wavelet transform knowledge representation (timefrequency) [16] in Fig. 10b and image knowledge representations (deduced from wavelet transformation) in Fig. 10c. For industrial diagnosis problems, the suggested hybrid intelligent diagnosis approach can be used. In fact, the same classification and decision-making processing architecture of the approach can be used with corresponding specific pertinent parameters and mainly modifications will be in PFS and FFS rule bases from specific experimental data.
Open Access This article is distributed under the terms of the Creative Commons Attribution License which permits any use, distribution, and reproduction in any medium, provided the original author(s) and the source are credited.

\section{References}

1. Balakrishnan, K., Honavar, V.: Intelligent Diagnosis Systems, Technical Report, Iowa State University, Ames, Iowa 50011-1040, U.S.A. (1997)

2. Turban, E., Aronson, J.E.: Decision Support Systems and Intelligent Systems, Int edn, 6th edn. Prentice-Hall (2001)

3. Karray, F.O., De Silva, C.: Soft Computing and Intelligent Systems Design, Theory, Tools and Applications. Addison Wesley, ISBN 0321-11617-8, Pearson Ed. Limited (2004) 
4. Meneganti, M., Saviello, F.S., Tagliaferri, R.: Fuzzy neural networks for classification and detection of anomalies. IEEE Trans. Neural Netw. 9(5), 848-861 (1998)

5. Palmero, G.I.S., Santamaria, J.J., de la Torre, E.J.M., Gonzalez, J.R.P.: Fault detection and fuzzy rule extraction in AC motors by a neuro-fuzzy ART-based system. Eng. Appl. Artif. Intell., 18, 867874 (2005) (Elsevier)

6. Piater, J.H., Stuchlik, F., von Specht, H., Mühler, R.: Fuzzy sets for feature identification in biomedical signals with self-assessment of reliability: an adaptable algorithm modeling human procedure in BAEP analysis. Comput. Biomed. Res. 28, 335-353 (1995)

7. Vuckovic, A., Radivojevic, V., Chen, A.C.N., Popovic, D.: Automatic recognition of alertness and drowsiness from EEG by an artificial neural network. Med. Eng. Phys., 24(5), 349-360 (2002)

8. Wolf, A., Barbosa, C.H., Monteiro, E.C., Vellasco, M.: Multiple MLP neural networks applied on the determination of segment limits in ECG signals. In: 7th International Work-Conference on Artificial and Natural NN, Proc. Part II, Menorca, Spain, June 2003, LNCS 2687, pp. 607-614. Springer, Berlin (2003)

9. Chohra, A., Kanaoui, N., Amarger, V.: A soft computing based approach using signal-to-image conversion for computer aided medical diagnosis (CAMD). In: Saeed, K., Pejas, J. (eds.) Information Processing and Security Systems, pp. 365-374. Springer, Berlin (2005)

10. Yan, H., Jiang, Y., Zheng, J., Peng, C., Li, Q.: A multilayer perceptron-based medical support system for heart disease diagnosis. Exp. Syst. Appl. (2005) (Elsevier)

11. Haykin, S.: Neural Networks: A Comprehensive Foundation, 2nd edn. Prentice-Hall (1999)

12. Zhang, G.P.: Neural networks for classification: a survey. IEEE Trans. Syst. Man Cybern. Part C Appl. Rev. 30(4), 451-462 (2000)
13. Egmont-Petersen, M., De Ridder, D., Handels, H.: Image processing with neural networks-a review. Pattern Recognit. 35, 2279$2301(2002)$

14. Don, M., Masuda, A., Nelson, R., Brackmann, D.: Successful detection of small acoustic tumors using the stacked derived-band auditory brain stem response amplitude. Am. J. Otol. 18(5), 608621 (1997)

15. Vannier, E., Adam, O., Motsch, J.F.: Objective detection of brainstem auditory evoked potentials with a priori information from higher presentation levels. Artif. Intell. Med. 25, 283-301 (2002)

16. Bradley, A.P., Wilson, W.J.: On wavelet analysis of auditory evoked potentials. Clin. Neurophysiol. 115, 1114-1128 (2004)

17. Azouaoui, O., Chohra, A.: Soft computing based pattern classifiers for the obstacle avoidance behavior of Intelligent Autonomous Vehicles (IAV). Int. J. Appl. Intell. 16(3), 249-271 (2002) (Kluwer Academic Publishers)

18. Zadeh, L.A.: Fuzzy sets. Inf. Control 8, 338-353 (1965)

19. Zadeh, L.A.: The calculus of fuzzy if / then rules. AI Expert, 23-27 (1992)

20. Lee, C.C.: Fuzzy logic in control systems: fuzzy logic controllerPart I \& Part II. IEEE Trans. Syst. Man Cybern. 20(2), 404-435 (1990)

21. Gonzalez, R.C., Woods, R.E.: Digital Image Processing, 2nd edn. Prentice-Hall (2002)

22. Farreny, H., Prade, H.: Tackling uncertainty and imprecision in robotics. In: 3rd International Symposium on Robotics Research, pp. 85-91 (1985) 